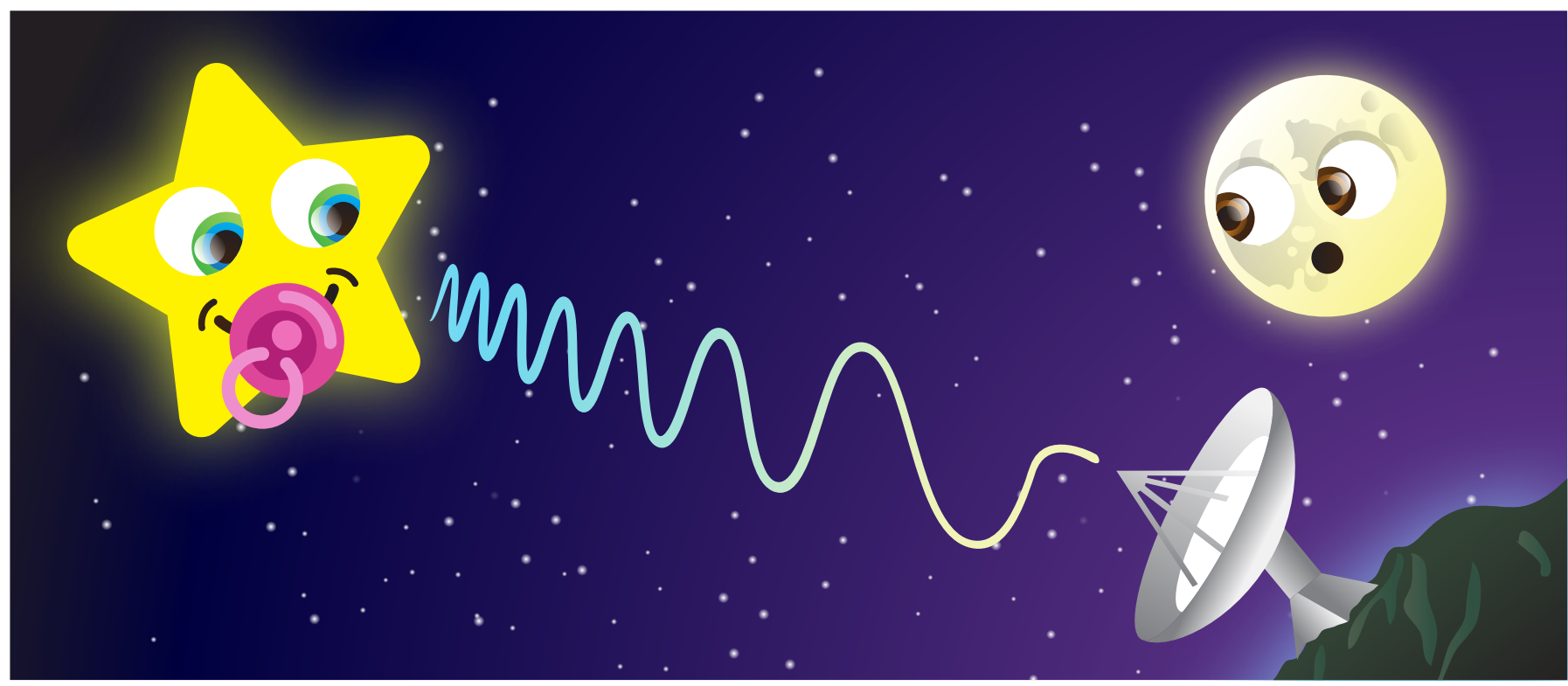

\title{
WHAT DO RADIO WAVES TELL US ABOUT THE
}

\section{UNIVERSE?}

\section{Sarah Scoles}

Science Journalist, Berkeley, CA, United States

\section{REVIEWED BY:}

GREEN BANK ELEMENTARY/ MIDDLE SCHOOL 11-12 YEARS OLD
Radio astronomy began in 1933 when an engineer named Karl Jansky accidentally discovered that radio waves come not just from inventions we create but also from natural stuff in space. Since then, astronomers have built better and better telescopes to find these cosmic radio waves and learn more about where they come from and what they can tell us about the universe. While scientists can learn a lot from the visible light they detect with regular telescopes, they can detect different objects and events - such as black holes, forming stars, planets in the process of being born, dying stars, and more - using radio telescopes. Together, telescopes that can see different kinds of waves - from radio waves to visible light waves to gamma rays - give a more complete picture of the universe than any one type of telescope can on its own.

When you look up at the night sky, you see the bright lights of stars. If you live in a dark place far from cities, you can see thousands of them. But the individual dots you see are all nearby stars. About 100 billion more stars, exist just in our galaxy, which is called the Milky Way. Beyond the Milky Way, astronomers 
ELECTROMAGNETIC

\section{SPECTRUM}

The visible light that we

can see is just a tiny part of the "electromagnetic spectrum." Visible light is made of photons with medium energy. Photons with more energy are ultraviolet radiation, $\mathrm{X}$-rays, and gamma rays (gamma rays have the most energy). Photons with less energy are infrared and radio waves (radio waves have the least energy).

\section{PHOTON}

Light is made of particles called photons, which travel in waves.

\section{WAVELENGTH}

The size of the wave a photon travels in.

\section{FREQUENCY}

The number of light waves that pass by a spot in one second.

think that about 100 billion more galaxies (each with their own 100 billion stars) exist. Almost all of these stars are invisible to your eyes, which cannot see the dim light from distant stars. Your eyes miss other things, too. The visible light that your eyes can see is only a tiny portion of what astronomers call the "electromagnetic spectrum," the whole range of different light waves that exists. The electromagnetic spectrum also includes gamma rays, $\mathrm{X}$-rays, ultraviolet radiation, infrared radiation, microwaves, and radio waves. Because human eyes can only see visible light, we have to build special telescopes to pick up the rest of that "spectrum" - and then turn them into pictures and graphs that we can see.

\section{WHAT IS A RADIO WAVE?}

Light is made up of tiny particles called "photons." Photons in visible light have a medium amount of energy. When photons have a little bit more energy, they become ultraviolet radiation, which you cannot see but which can give you a sunburn. With more energy than that, photons become X-rays, which travel right through you. If photons possess even more energy, they become gamma rays, which come out of stars when they explode.

But when photons have a little less energy than visible-light photons, they are known as infrared radiation. You can feel them as heat. Finally, we call the photons with the least energy "radio waves." Radio waves come from strange spots in space - the coldest and oldest places and the stars with the most material stuffed into a small space. Radio waves tell us about parts of the universe we would not even know existed if we only used our eyes or telescopes that see visible photons.

\section{WAVELENGTH AND FREQUENCY}

Radio astronomers use these radio photons to learn about the invisible universe. Photons travel in waves, like they are riding a roller coaster that just uses the same two pieces of track over and over [1]. The size of a photon's wave - its wavelength - tells you about its energy. Figure 1 shows waves with two different wavelengths. If the wave is long, it does not have much energy; if it is short, it has a lot of energy. Radio waves do not have much energy, and that means they travel in big waves with long wavelengths. Radio waves can be hundreds of feet across or just a few centimeters across.

Astronomers also talk about how many of these waves pass a spot every second - the radio wave's "frequency." You can think of frequency by imagining a pond of water. If you throw a rock into the water, ripples travel across 


\section{FIGURE 1}

Photons travel in waves. The length of each wave is called a wavelength.

\section{HERTZ}

$1 \mathrm{~Hz}$ means that one wave passes by a spot in one second. One megahertz means one million waves pass by every second.

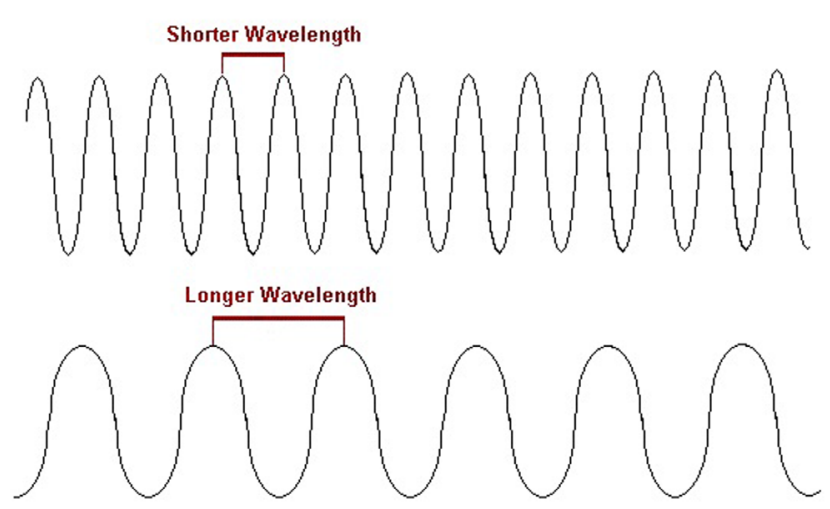

FIGURE 1

the pond. If you stand in the water, the waves hit your ankles. The number of waves that smack into you in one second tells you the frequency of the waves. One wave per second is called 1 Hertz. A million waves per second is $1 \mathrm{MHz}$. If the waves are long, fewer of them hit you every second, so long waves have smaller frequencies. Radio waves have long wavelengths and small frequencies.

\section{RADIO PIONEERS}

The first radio astronomer did not mean to be the first radio astronomer. In 1933, a man named Karl Jansky was working on a project for Bell Laboratories, a lab in New Jersey named after Alexander Graham Bell, who invented the telephone. Engineers there were developing the first phone system that worked across the Atlantic Ocean. When people first tried making phone calls on that system, they heard a hissing sound in the background at certain times of the day. Bell Labs thought that noise was bad for business, so they sent Karl Jansky to find out what was causing it. He soon noticed that the hiss began when the middle of our galaxy rose in the sky and ended when it set (everything in the sky rises and sets just like the Sun and Moon do). He figured out that radio waves coming from the center of the galaxy were messing up the phone connection and causing the hiss. $\mathrm{He}$ - and the phone - had detected radio waves from space [1]. Jansky opened up a new, invisible universe. You can see a picture of the antenna used by Karl Jansky to detect radio waves from space in Figure 2.

Inspired by Janksy's research, a man named Grote Reber built a radio telescope in his backyard in Illinois. He finished the telescope, which was $31 \mathrm{ft}$ across, in 1937 and used it to look at the whole sky and see where radio waves came from. Then, from the data he collected from his radio telescope, he made the first map of the "radio sky" [2]. 


\section{FIGURE 2}

The founder of radio astronomy, Karl Jansky, stands with the antenna he built that detected the first radio waves identified as coming from space. Source: NRAO.

\section{FIGURE 3}

While instruments like the Green Bank Telescope, pictured here, may not look like traditional telescopes, they work much the same way but detect radio waves instead of visible light. They then turn those radio waves, which human eyes cannot see, into pictures and graphs that scientists can interpret. Source: NRAO.

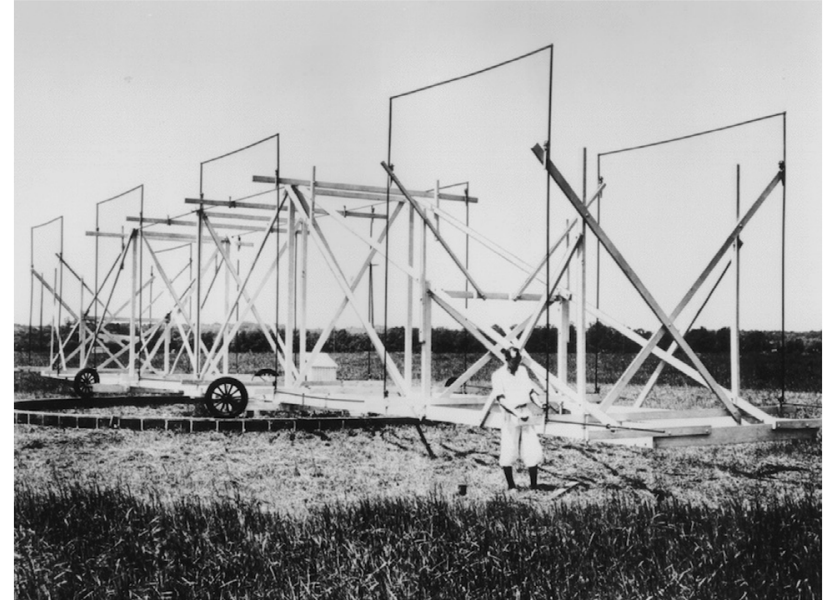

FIGURE 2

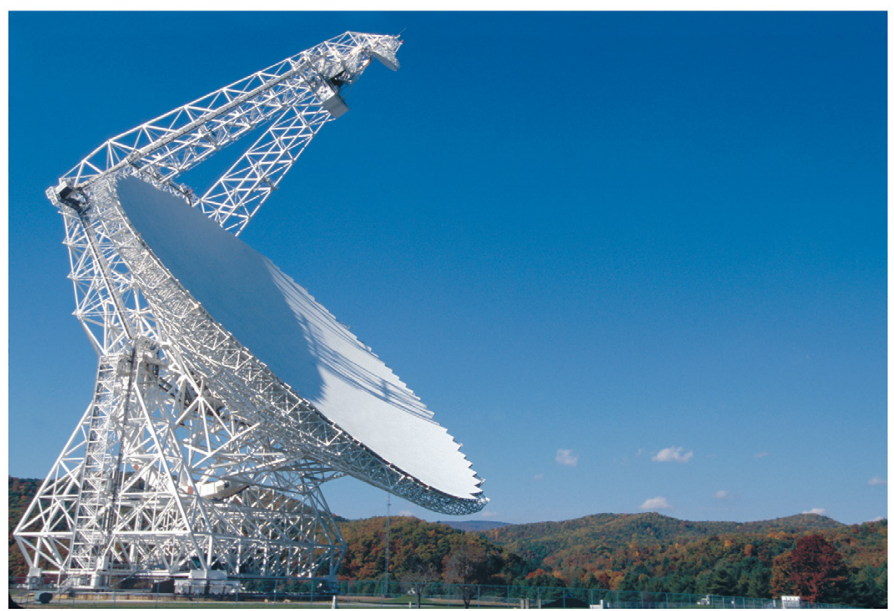

FIGURE 3

\section{RADIO TELESCOPE TALK}

You can see visible light because the visible-light photons travel in small waves, and your eye is small. But because radio waves are big, your eye would need to be big to detect them. So while regular telescopes are a few inches or feet across, radio telescopes are much larger. The Green Bank Telescope in West Virginia is more than $300 \mathrm{ft}$ wide and can be seen in Figure 3. The Arecibo Telescope in the jungle in Puerto Rico is almost $1,000 \mathrm{ft}$ across. They look like gigantic versions of satellite TV dishes, but they work like regular telescopes.

To use a regular telescope, you point it at an object in space. Light from that object then hits a mirror or lens, which bounces that light to another mirror or lens, which then bounces the light again and sends it to your eye or a camera.

When an astronomer points a radio telescope at something in space, radio waves from space hit the telescope's surface. The surface - which may be 


\section{RECEIVER}

The part of a radio telescope that takes the radio waves and turns them into a picture.

metal with holes in it, called mesh, or solid metal, like aluminum - acts like a mirror for radio waves. It bounces them up to a second "radio mirror," which then bounces them into what astronomers call a "receiver." The receiver does what a camera does: it turns the radio waves into a picture. This picture shows how strong the radio waves are and where they are coming from in the sky.

\section{RADIO VISION}

When astronomers look for radio waves, they see different objects and events than they see when they look for visible light. Places that seem dark to our eyes, or to regular telescopes, burn bright in radio waves. Places where stars form, for example, are full of dust. That dust blocks the light from getting to us, so the whole area looks like a black blob. But when astronomer turns radio telescopes to that spot, they can see straight through the dust: they can see a star being born.

Stars are born in giant clouds of gas in space. First, that gas clumps together. Then, because of gravity, more and more gas is attracted to the clump. The clump grows bigger and bigger and hotter and hotter. When it is huge and hot enough, it starts smashing hydrogen atoms, the smallest atoms that exist, together. When hydrogen atoms crash into each other, they make helium, a slightly bigger atom. Then, this clump of gas becomes an official star. Radio telescopes take pictures of these baby stars [3].

Radio telescopes show the secrets of the nearest star, too. The light we see from the Sun comes from near the surface, which is about $9,000^{\circ} \mathrm{F}$. But above the surface, the temperature reaches $100,000^{\circ} \mathrm{F}$. Radio telescopes help us learn more about these hot parts, which send out radio waves.

The planets in our solar system also have radio personalities. Radio telescopes show us the gases that swirl around Uranus and Neptune and how they move around. Jupiter's north and south poles light up in radio waves. If we send radio waves toward Mercury, and then catch the radio waves that bounce back using a radio telescope, we can make a map almost as good as Google Earth [4].

When they look much farther away, radio telescopes show us some of the weirdest objects in the universe. Most galaxies have supermassive black holes in their centers. Black holes are objects that have a lot of mass squished into a tiny space. This mass gives them so much gravity that nothing, not even light, can escape their pull. These black holes swallow stars, gas, and anything else that comes too close. When that unlucky stuff feels the black hole's gravity, it first spirals around the black hole. As it gets closer, it goes faster and faster. Huge jets, or columns, of electromagnetic radiation and matter that does not make it in to the black hole (sometimes taller than a whole galaxy is wide) 


\section{FIGURE 4}

Galaxies that have supermassive black holes at their centers can shoot out jets of material and radiation, like those seen here, that are taller than the galaxy is wide.

Source: NRAO.

\section{DARK ENERGY}

Dark energy acts like the opposite of gravity and pushes everything in the universe farther apart.

\section{MEGAMASER}

A natural laser in space that sends out radio waves, instead of red or green light like the kind that comes from a laser pointer.

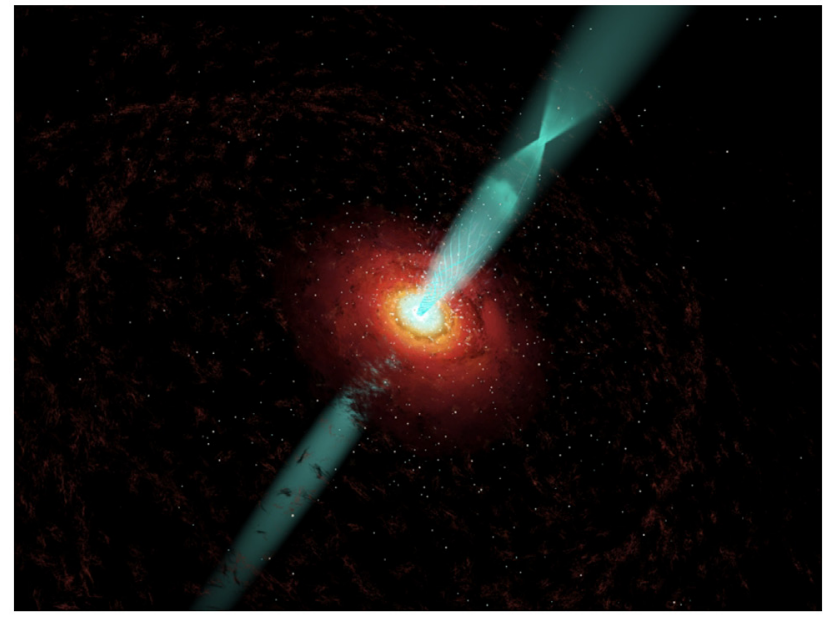

form above and below the black hole. Radio telescopes show those jets in action (Figure 4).

Massive objects like these black holes warp the fabric of space, called spacetime. Imagine setting a bowling ball, which weighs a lot, on a trampoline. The trampoline sags down. Weighty stuff in space makes space-time sag just like the trampoline. When radio waves coming from distant galaxies travel over that sag to get to Earth, the shape acts just like the shape of a magnifying glass on Earth: telescopes then see a bigger, brighter picture of the distant galaxy.

Radio telescopes also help solve one of the biggest mysteries in the universe: What is dark energy? The universe is getting larger every second. And it gets larger faster and faster every second because "dark energy" is the opposite of gravity: Instead of pulling everything together, it pushes everything farther apart. But how strong is dark energy? Radio telescopes can help scientists to answer this question by looking at "megamasers" that occur naturally in some parts of space, a megamaser is kind of like a laser on Earth, but it sends out radio waves instead of the red or green light that we can see. Scientists can use megamasers to pin down the details of dark energy [5]. If scientists can figure out how far away those megamasers are, they can tell how far away different galaxies are, and then they can figure out how fast those galaxies are speeding away from us.

\section{A FULL TOOLBOX}

If we only had telescopes that picked up visible light, we would be missing out on much of the action in the universe. Imagine if doctors had only a stethoscope as a tool. They could learn a lot about the patient's heartbeat. But they could learn so much more if they also had an X-ray machine, a sonogram, an 
MRI instrument, and a CT scanner. With those tools, they could get a more complete picture of what was happening inside the patient's body. Astronomers use radio telescopes together with ultraviolet, infrared, optical, X-ray, and gamma-ray telescopes for the same reason: to get a complete picture of what is happening in the universe.

\section{REFERENCES}

1. Jansky, K. G. 1993. Radio waves from outside the solar system. Nature 32, 66. doi: 10.1038/132066a0

2. Reber, G. 1944. Cosmic static. Astrophys. J. 100, 297. doi: 10.1086/144668

3. McKee, C. F., and Ostriker, E. 2007. Theory of star formation. Annu. Rev. Astron. Astrophys. 45, 565-687. doi: 10.1146/annurev.astro.45.051806.110602

4. Ostro, S. J. 1993. Planetary radar astronomy. Rev. Mod. Phys. 65, 1235-79. doi: 10.1103/RevModPhys.65.1235

5. Henkel, C., Braatz, J. A., Reid, M. J., Condon, J. J., Lo, K. Y., Impellizzeri, C. M. V., et al. 2012. Cosmology and the Hubble constant: on the megamaser cosmology project (MCP). IAU Symp. 287, 301. doi: 10.1017/S1743921312007223

SUBMITTED: 19 August 2015; ACCEPTED: 15 January 2016;

PUBLISHED ONLINE: 03 February 2016.

EDITED BY: Kathryn Elizabeth Williamson, National Radio Astronomy Observatory, United States

CITATION: Scoles S (2016) What do radio waves tell us about the universe? Front. Young Minds 4:2. doi:10.3389/frym.2016.00002

COPYRIGHT () 2016 Scoles. This is an open-access article distributed under the terms of the Creative Commons Attribution License (CC BY). The use, distribution or reproduction in other forums is permitted, provided the original author(s) or licensor are credited and that the original publication in this journal is cited, in accordance with accepted academic practice. No use, distribution or reproduction is permitted which does not comply with these terms.

\section{REVIEWED BY:}

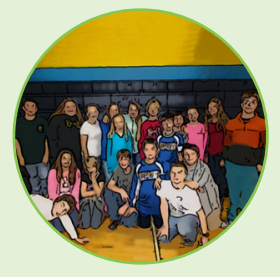

\section{GREEN BANK ELEMENTARY/MIDDLE SCHOOL, $11-12$ YEARS OLD}

We are the sixth grade class at our school. There is only one sixth grade this year, and we are it. We are 23 of us and most of us have known each other since kindergarten. We had a scientist come from the observatory to tell us about space. She came twice, and we really liked it. Science is a favorite subjects for many of us in the class. We also like that Mrs. Tallman, our English/Language Arts teacher and Ms. Smith, our Science teacher, agreed to help us do this project. Other things we like to do are: hunting, fishing, riding four wheelers, basketball, football, soccer, baseball and softball, math class, playing games on the computer, and playing with our friends. We also like to talk. 


\section{AUTHOR}

\section{SARAH SCOLES}

I am a science writer my work has appeared in The Atlantic, Slate, The Guardian, Popular Science, and others. Previously, I worked as an editor at Astronomy magazine and as a public educator at the National Radio Astronomy Observatory in Green Bank, WV, United States home to one of the world's largest radio telescopes. *sarah.scoles@gmail.com 